\title{
SEMIOTIQUE DES CULTURES ET SCIENCES DE LA CULTURE CHEZ FRANÇOIS RASTIER \\ SEMIÓTICA DAS CULTURAS E CIÊNCIAS DA CULTURA EM FRANÇOIS RASTIER
}

\author{
Driss EL KHATTAB \\ Faculté des Lettres, Mohammedia \\ Casablanca - Marrocos \\ Khattab dr@yahoo.fr
}

RÉSUMÉ. L'objet de cette contribution est l'opposition entre «sciences de la culture» et «sémiotique des cultures». Nous tenterons de mettre en exergue la position de François Rastier quant aux aspects épistémologiques et méthodologiques de ces deux types de savoirs en sciences humaines et sociales. Les deux objets visant la culture ont un programme unificateur et jouissent d'une validité épistémologique. Il s'avère que la vocation fédératrice revient aux sciences de la culture et que la définition d'une perspective proprement sémiotique sur la culture est un chantier de la recherche dans l'avenir.

Mots-clés : Sémiotique. Sciences de la culture. Épistémologie. Méthodologie. Fédération. Unification. Disciplines. Texte. Sens.

RESUMO. O objeto dessa contribuição é a oposição entre «ciências da cultura» e «semiótica das culturas». Procuraremos destacar a posição de François Rastier sobre os aspectos epistemológicos e metodológicos desses dois tipos de conhecimento nas ciências humanas e sociais. Ambos os objetos para cultura têm um programa unificador e têm validade epistemológica. Acontece que a vocação unificadora remonta às ciências da cultura e que a definição de uma perspectiva propriamente semiótica da cultura é um local de pesquisa no futuro.

Palavras-chave: Semiótica. Ciências da cultura. Epistemologia. Metodologia. Federação. Unificação. Disciplinas. Texto. Significado.

\section{Introduction}

La sémiotique a connu un développement notable, qui s'est effectué par le passage d'une sémiotique du signe à une sémiotique du texte. Par la suite, elle a tenté de rendre compte de la culture. Cependant, un autre courant de pensée d'est constitué depuis le $18 \mathrm{e}$ siècle et proclamait que son objet de recherche est l'ensemble des faits de cultures et leurs diverses manifestations dans les sociétés humaines; il s'agit des sciences de la culture. Dans cette communication, nous tenterons de mettre en exergue la position de François Rastier quant aux aspects épistémologiques et méthodologiques de ces deux types de savoirs en sciences humaines et sociales. Nous examinons le programme fédérateur des sciences de la 
culture et celui de la sémiotique des cultures. Lequel des deux jouit d'une validité épistémologique?

\section{Sémiotique des cultures : objet et méthodes}

La sémiotique des cultures ou de la culture est un courant de pensée qui a mis en place deux approches qui se sont caractérisées par une grande production scientifique, par des propositions intéressantes dans l'essai d'analyse des objets culturels. Ces deux approches sont constituées par l'Ecole de Moscou-Tartu et l'Ecole de Paris. La première approche tend à relier la sémiotique à la littérature comparée et s'évertue à analyser les textes en tenant compte des cultures spécifiques comme la culture russe. La seconde approche privilégie la linguistique, conçue comme étant un modèle d'analyse qui permet de relier les textes aux cultures. Cette approche est représentée par Greimas, Rastier, Fontanille et d'autres. Nous mettons l'accent sur l'Ecole française, et nous examinons deux questions épistémologiques : la première concerne les objets problématiques de la sémiotique des cultures chez Rastier et la seconde traite de la question de l'opposition entre les notions "sémiotique des cultures» et "sciences de la culture ».

Il est possible de distinguer trois conceptions qui sont rattachées à la notion de sémiotique des cultures. La première conception est intégrée dans la sphère de la sémiotique générale ou discursive, Greimas (1966) apparaît comme étant le représentant de ce courant qui postule que l'objet de la sémiotique est un objet empirique qui est formé d'un large éventail de textes et des cultures qui les englobent. La culture apparaît ainsi comme un facteur dissolu dans le texte. Dans cette perspective, Greimas (1979) admet que les formes sémiotiques sont des formes signifiantes. Dans son approche du texte, le principe d'immanence est un principe ouvert, qui permet d'étudier le texte en lui-même, mais aussi montrer ses liens avec la culture ambiante, rendant possible la constitution d'une sémiotique des cultures . En outre, Greimas (1984) déclare que l'objet de la sémiotique est la culture en général, mais son objet reste indéfini. Nous en déduisons que les faits culturels sont inscrits dans le texte sous forme de modèles conceptuels et idéologiques. 
La deuxième conception est développée par Jaques Fontanille $(2004,2013)$ qui tente de fonder une sémiotique des cultures sur la base d'une hiérarchie des signes et propose de ce fait ses analyses à partir de notions théoriques, telles que celle de «processus » et de « niveau de pertinence sémiotique ». Il semble que dans cette mouvance, la pertinence est le concept central. Fontanille (2004) met en place une approche qui est issue de ses travaux effectués jadis en collaboration avec Greimas. La sémiotique envisagée reflète en quelque sorte le parcours des préoccupations successives de deux ou trois générations de sémioticiens. L'auteur se distingue en outre par des innovations théoriques et méthodologiques qui tendent à rendre compte des faits culturels dans la société moderne. Ce parcours l'a conduit à établir un ensemble de distinctions fondatrices d'une sémiotique des cultures. Dans cette perspective, il prévoit des niveaux d'analyse afin d'établir une hiérarchie méthodologique de l'analyse et de la perception des objets constitutifs de la culture. ${ }^{1}$ Mais, le modèle ci-dessus se limite à la description des articulations des performances sémiotiques; il ne propose pas une théorie complète et indépendante. Nous estimons que la sémiotique des cultures envisagée par Fontanille est une sémiotique générale alimentée par des éléments analytiques des sémiotiques particulières comme celles de la musique, des sensations, du goût, etc.

Quant à la troisième conception, elle est promue par F.Rastier qui, partant du postulat que la linguistique est une sémiotique des langues, considère que la sémantique des textes ou sémantique textuelle est le cadre proposé pour la description et l'analyse des textes de tout genre. F.Rastier (1996,2001a, 2002a) soutient l'hypothèse que la linguistique est devenue le noyau fondateur de la sémiotique moderne, à travers son projet scientifique qui la sépare de la philosophie et moyennant son épistémologie issue des sciences historiques et

\footnotetext{
1. Fontanille (2004, p.2) distingue de ce fait « deux niveaux d'expérience dont découlent deux types d'entités pertinentes: l'expérience figurative (et iconique) d'un côté, dont on extrait comme grandeurs pertinentes de l'expression des signes, et, de l'autre côté, l'expérience textuelle (et intentionnelle-interprétative), dont on extrait comme grandeurs pertinentes de l'expression des textes-énoncés». L'auteur a ensuite établi six niveaux qui lui semblent pertinents dans la sémiotique des cultures, ces niveaux sont : les signes ou figures, les textes-énoncés, les objets, les scènes et pratiques, les stratégies, et les formes de vie ; à chaque niveau, le principe de pertinence distingue une instance formelle-structurelle et une instance matérielle sensible » (2004,p. 14). Cet arsenal conceptuel et méthodologique est minutieusement présenté pour opérer les commutations, les segmentations et les catalyses, opérations nécessaires pour dégager les signifiés et les valeurs.
} 
comparatives. L'auteur admet que la sémiotique des cultures est le domaine du savoir qui est fondé par des linguistes tels que Saussure et Hjelmslev, et qui relie les caractéristiques des langues à celles des systèmes de signes et des formations culturelles que Cassirer a nommé «les formes symboliques ». Contrairement à Greimas et Fontanille, l'approche de Rastier se distingue par l'élaboration d'une théorie sémantique des textes et des performances sémiotiques et par la redéfinition des concepts théoriques et méthodologiques. Le modèle envisagé par l'auteur dans de nombreux écrits, notamment ses ouvrages sens et textualité et Arts et sciences du texte, est basé sur une théorie sémantique qui conduit au projet des sciences de la culture.

Rappelons que la sémiotique traite, entre autres, des objets suivants : a- le système de signes non linguistiques, b- l'ensemble des principes communs aux langues et aux systèmes de signes non linguistiques (Hjelmslev, Greimas), cl'étude de la manière dont le monde fait signe (Eco), d- l'étude du monde animale 2. La rupture avec les approches formalistes telles qu'elles sont vilipendées par Greimas était l'événement qui a amené la sémiotique à s'orienter vers l'étude des signes culturels. En réfutant les approches réductionnistes qui sont centrées sur le signe, Rastier a proposé la théorie de la sémantique des textes (2001a) dans laquelle il a spécifié la relation entre le sens et le signe ${ }^{3}$.

Dans son ouvrage Arts et sciences du texte, Rastier a critiqué la sémiotique telle qu'elle est conçue par Greimas, il a constaté qu'elle n'a pas pu élaborer un appareil théorique propre aux textes, et de surcroît, elle n'a pas pu dépasser le stade du texte. L'auteur s'est évertué à démontrer les limites de ses mécanismes d'analyse, fondés principalement sur le concept de parcours génératif qui déduit le

\footnotetext{
2. Rastier, (1996, p.48-49).

3. Dans cette perspective, Rastier (2001a) a opéré une redéfinition des concepts fondateurs de la sémiotique, et considère que le signe ne peut être l'objet d'interprétation du fait qu'il est coupé de ses contextes. L'auteur souligne en effet que «le signe ne peut être interprété, puisque l'isoler le coupe précisément de ses conditions d'interprétation et de son contexte» (2001a, p.103). Il considère en outre que la sémiosis n'est pas la relation qui s'instaure entre signifiant et signifié, et le signifiant n'est pas le point de départ dans la recherche sémiotique. Il précise que la sémiosis , qui est défini grâce à la notion de parcours interprétatif, «doit être rapportée aux deux plans du contenu et de l'expression des textes et des autres performances sémiotiques» (Ibid.p.103). Dans cette approche, «les relations constituantes du sens vont de signifiés en signifiés, mais aussi des signifiés vers les signifiant: ainsi, la sémiosis se définit comme un réseau des relations entre signifiés au sein du texte, en considérant les signifiants comme des interprétants qui permettent de construire certaines de ces relations » (ibid. p.103-104).
} 
sens textuel de la signification"; la sémiotique est amenée, à ses yeux, à produire des théories du sens, car, jusqu'à présent, elle n'a mis en place que des théories de la signification. De manière générale, la sémiotique contemporaine n'a pas produit de théorie de texte compatible avec une problématique rhétorique herméneutique ${ }^{5}$. L'auteur a proposé en contrepartie la sémantique des textes, qui est intégrée dans le paradigme rhétorique-herméneutique. Ainsi, la pensée de Rastier peut se résumer ainsi: la théorie de la sémantique des textes fait partie de la sémiotique des cultures qui aboutit in fine aux sciences de la culture.

Sur le plan épistémologique, Rastier (2001a) remet en question le statut de la sémiotique en considérant que «l'omniprésence des signes rend impossible sa constitution de la sémiotique comme discipline» ${ }^{6}$. La sémiotique des cultures, elle aussi «ne s'est pas constituée en discipline autonome $»^{7}$. Ces affirmations conduisent l'auteur à ne garder de cette dernière qu'une vocation épistémologique: "fédérer les sciences de la culture autour des concepts de langage et d'interprétation $»^{8}$. Une telle vocation met la sémiotique de la culture en concurrence avec les sciences de la culture qui, elles aussi, fédèrent les sciences humaines et sociales en général et les faits de culture en particulier. Avant de poursuivre notre analyse des faits épistémologiques concernant le statut de la sémiotique, nous présentons, ne serait ce que sommairement, les caractéristiques générales des sciences de la culture. Quel est l'objet de ces savoirs? Et quels sont leurs rapports avec la sémiotique des cultures?

\section{Les sciences de la culture : objet, méthodes et objectifs}

Les sciences de la culture est un projet scientifique qui est apparu à la fin du $18^{e}$ siècle, et s'est affermi avec la disciplinarisation qui a suivi l'essor des

\footnotetext{
4 «le sens n'est pas donné par un codage préalable qui associerait strictement un signifiant et un signifié ou une classes de signifiés ( car la langue n'est pas une nomenclature) : il est produit dans des parcours qui discrétisent et unissent des signifiés entre eux, en passant par des signifiés » ibid. p.104.

${ }^{5}$ Rastier, (1996, p.39).

${ }^{6}$ Rastier, (2001a, p.282).

7 ibid. p.283.

8 ibid. p. 284.
} 
universités au $19^{\mathrm{e}}$ siècle. Trois significations peuvent être accordées à cette notion ${ }^{9}$ :

(i) Les sciences de la culture réfèrent aux recherches en sciences humaines qui traitent de l'esprit ;

(ii) Les sciences de la culture sont focalisées sur les recherches qui s'appuient sur les méthodes scientifiques pour rendre compte des faits de culture, il s'agit des disciplines comme la linguistique, l'anthropologie, la paléontologie, etc. ;

(iii) Elles constituent une synthèse entre les sciences humaines et sociales d'une part et les sciences de la vie et de la nature d'autre part, elles ambitionnent même d'imiter les sciences exactes et aspirent à les unifier pour rendre compte des performances sémiotiques de l'être humain.

La première conception est globalisante et elle est fondée sur des considérations philosophiques. De plus, la culture est un concept général qui est étudié par des philosophes et épistémologues en termes de la démarche classique de la philosophie. La seconde conception est réductionniste, en ce sens que les disciplines qui en rendent compte se focalisent, tradition scientifique oblige, sur un aspect de la culture: la langue pour la linguistique, les rites, les croyances, la manière de vivre des ethnies pour l'anthropologie, l'environnement des hommes anciens pour la paléontologie, etc. alors que dans la dernière acception qui a retenu notre attention et qui fait le thème central d'un travail antérieur ${ }^{10}$, les sciences de la culture se caractérisent par l'extension de ses objets d'étude, la multitude des disciplines sollicitées, et la difficulté à déterminer son identité. Elles se caractérisent notamment par le dépassement de la fausse distinction entre sciences humaines et sciences sociales. Dans ce contexte, nombre de champs disciplinaires ont présenté des projets englobants, il s'agit de la sociologie de Durkheim, la sémiologie de Ferdinand de Saussure, la sémiotique de la culture de Lotman et la sémiotique des cultures de Rastier. Dans un souci de compromis, Rastier appelle de ses vœux à la voie fédérative qui assure une épistémologie de complémentarité entre les sciences de la nature, de la vie et les sciences de la culture. La position de Rastier consiste à défendre la «voie fédérative», qui est, à ses yeux, la seule perspective globale capable de s'opposer au computationnalisme.

\footnotetext{
${ }^{9}$ Cf. El Khattab, (2017, chap. I).

10 Nous référons à notre dernier ouvrage intitulé «Les sciences de la culture» paru en arabe chez Toubkal, 2017, Casablanca, Maroc.
} 
Face aux sciences cognitives qui entendent naturaliser les phénomènes culturels, les sciences de la culture proposent des programmes pluridisciplinaires pour rendre compte des faits culturels les plus variés. En effet, «Les sciences de la culture sont les seules à pouvoir rendre compte du caractère sémiotique de l'univers humain. Pour connaître l'humain par l'homme, elles doivent reconnaître la part qu'il prend dans cette connaissance, non seulement comme destinataire critique de «résultats», mais comme acteur doué d'affects et de responsabilité» ${ }^{11}$.

Comme on pourrait le constater, la sémiotique des cultures et les sciences de la culture telles quelles sont présentées plus haut constituent deux types de savoirs distincts ; cependant, les sciences de la culture ont le statut d'un ensemble de savoirs dont la sémiotique n'est qu'une partie. Cette problématique fera l'objet de la section suivante.

\section{Problématique}

Dans l'optique de Rastier (2001), sémiotique des cultures et sciences de la culture rivalisent en ce qui concerne leur objet: les faits de culture sous ses différentes formes dans les sociétés humaines. Alors pourquoi maintient-on les deux expressions, «sémiotique des cultures» et «sciences des cultures»? Est-ce que la sémiotique des cultures est une discipline? A-t-elle le statut d'une science ou des sciences?

Rastier (2002a, p.8) affirme que :

«une sémiotique des cultures ne serait pas à vrai dire une discipline, mais le projet même de redéfinir la spécificité des sciences sociales: les cultures ambrassent la totalité des faits humains, jusqu'à la formation des sujets» ${ }^{12}$.

Dans cette perspective, nous sommes devant deux types de savoirs qui se distinguent par le refus de la spécialisation et qui se positionnent en tant que projets scientifiques dont la fonction est de fédérer les sciences sociales et les faits de culture. Ainsi, il semble nécessaire de préciser la l'emploi des deux expressions. L'auteur remarque que la notion de «sciences de la culture» est empruntée à Cassirer (1942), et celle de «sémiotique des cultures» renvoie à l'Ecole de Tartu-

\footnotetext{
${ }^{11}$ Rastier, (2002a, p.4).

12 .Rastier,( 2002a, p.8).
} 
Moscou $^{13}$. Dans un article paru dans la revue Linx (2001), Rastier pose la question du statut des deux savoirs, et examine la dualité terminologique. La problématique qui s'en suit s'articule autour de deux conceptions:

1. La sémiotique des cultures est une partie de la sémiotique générale. Elle intéresse foncièrement les textes suivant les principes méthodologiques mis en place par la sémantique des textes. Quant elle poursuit un objectif de caractérisation, la sémiotique des cultures a le statut d'un champ de savoirs dérivé de la linguistique. L'auteur s'est positionné par rapport au grand nombre d'approches et de théories sémiotiques. Il a ainsi adopté la méthodologie de perception du sens à travers les corpus, refuse les sémiotiques universelles et la philosophie du sens. Pour rendre compte des cultures, il tend à promouvoir la sémiotique comparative et différentielle.

2. La sémiotique des cultures est un projet global et plus général que les sciences de la culture, du moment que sa fonction épistémologique consiste à fédérer les sciences de la culture. C'est dans cette perspective qu'elle n'est pas conçue en tant que discipline.

Force est de constater que cette même fonction soulignée dans (2) a été observée chez Lotman (1993) qui a proposé un projet épistémologique fondé sur la base de l'interdisciplinarité et de la mise en place des liens entre différents champs des sciences humaines et sociales. Conçue comme étant la science des sciences, la sémiotique des cultures assumera la fonction de regroupement des disciplines des sciences humaines et sociales, sans les unifier, exercera un contrôle critique sur le caractère culturel de ses objets, et déterminera en quelques sortes leurs objectifs. Une telle sémiotique n'est pas une discipline, car elle est dépourvue des critères d'évaluation, de plus elle se caractérise par son ouverture à divers domaines des sciences humaines et sociales. «Elle s'ouvre ainsi d'une part à l'éthologie des sociétés humaines, de l'autre sur une philosophie es formes symboliques» ${ }^{14}$. Cette argumentation conduit à déduire que la sémiotique des cultures est une sémiotique générale, qui définit le champ où la linguistique,

${ }^{13}$ Cf. Rastier, (2002a, p.4). 
l'iconologie, la musicologie et les autres sciences sémiotiques entretiennent des relations étroites en termes de méthode de pluridisciplinarité. Ainsi, la pluridisciplinarité constitue un tournant dans la méthodologie contemporaine des sciences humaines et sociales. Fédérer ou unifier les sciences et les approches paraît une entreprise attractive, mais quelle est la place de la sémiotique des cultures dans cette entreprise? Et comment résoudre la problématique signalée plus haut?

\section{La sémiotique fédérative}

L' hypothèse de la sémiotique fédérative stipule que celle-ci subsume les sciences de la culture et assume à leurs égards des responsabilités comme celle du contrôle critique des savoirs qui visent les cultures. Rastier (200a) avance deux arguments en faveur de cette hypothèse :

1- Le programme des sciences de la culture est déjà intégré dans quelques disciplines en sciences humaines et sociales. En effet, l'auteur remarque que leur programme trouve son origine dans le programme anthropologique de Humboldt d'une part, et dans la recherche sémiotique, d'autre part. Dans le premier cas, il est fondé sur la caractérisation des groupes humains et des langues ( Rastier, 2002a,p.4) ; dans l'autre, sur l'omniprésence des signes et leur diversité dans le monde sémiotique ${ }^{15}$;

2- «C'est la reconnaissance de la spécificité et de l'autonomie relative du monde sémiotique qui permet de délimiter le champ des sciences de la culture» ${ }^{16}$.

Ces hypothèses ont suscité des discussions dont l'importance mérite d'être restituée dans cette communication. En effet, Fontanille (2004) estime que d'autres savoirs rattachés aux sciences humaines et sociales peuvent assumer la fonction fédératrice que Rastier (2001, 2001a, 2002a) tend à accorder à la

\footnotetext{
${ }^{15}$ Rastier souligne l'importance des faits sémiotiques dans la caractérisation de l'espèce humaine opposé à l'espèce animale, dans ces termes: "L'innovation et sa transmission ne suffisent pas à définir la spécificité des cultures humaines; c'est la diversification et l'autoréflexion des pratiques techniques et sémiotiques qui les distingue », (Rastier, 2002a,p. 5).

${ }^{16}$ Rastier, (2002a, p.9).
} 
sémiotique des cultures. L'auteur a évoqué la sociologie des sciences, la philosophie des savoirs et la psychologie cognitive. Selon lui, la sémiotique générale peut opposer quelques propositions complémentaires. On peut, dit-il, fédérer les sciences de la culture de deux manières : a) en élaborant une définition claire et précise des phénomènes culturels qui embrassent tous les objets produits par l'activité humaine. L'auteur rappelle que l'Ecole de Tartu était le précurseur en ce qui concerne la proposition d'un modèle de sémiotique général des faits culturels, intégrant toues les sciences sociales. Ce modèle est fondé sur le principe de sémiosphère. b) Il y a lieu de fédérer non seulement les pratiques d'analyse des objets culturels, mais aussi les types d'objets culturels, définis par leurs propriétés sémiotiques discursives. Ces propriétés, estime Fontanille (2013), se présentent comme des classes d'expérience culturelle correspondant à des niveaux de phénoménalité. Fontanille reproche à Rastier le fait de limiter sa sémiotique aux textes et de ne pas hiérarchiser les objets culturels. C'est la raison pour laquelle il indique, en termes d'argumentation, que les sémiotiques-objets constitutives des cultures sont diverses, et fait allusion aux types sémiotiques qu'il a étudiés: les figures-signes, les textes-énoncés, les objets-supports, les pratiques, les stratégies et les formes de vie.

Mais les sciences de la culture sont des savoirs globalisants et subsument les différentes disciplines ou corps de savoirs évoqués par Fontanille. Il faut examiner les spécificités des sciences de la culture pour s'en rendre compte. La section ci-après traite de cette question qui est encore à ses balbutiements.

\section{Spécificités des sciences de la culture}

Les sciences de la culture sont évoquées dans les premiers écrits des philosophes de la première moitié du 20 e siècle, il s'agit d'approches épistémologiques (Dilthey, Cassirer, Weber, notamment) qui s'évertuent à mettre en lumière les caractéristiques saillantes des sciences de la culture par rapport aux sciences de la nature. En effet, ces auteurs soulignent que a) les sciences de la culture appartiennent au champ de la critique historique, b) elles ont le statut de sciences herméneutiques ou interprétatives, c) elles sont disposées à rendre compte sur le plan méthodologique de la description des phénomènes humains et 
sociaux, d) elles se caractérisent par la rigueur de leurs méthode, e) elles constituent des sciences idiographiques et non nomothétiques, du fait qu'elles visent, entre autres, la singularité des objets représentés notamment par l'œuvre d'art non reproductible, f) elles sont des sciences des valeurs et non des faits ${ }^{17}$.

Dans l'œuvre de Rastier, l'accent est mis sur la complexité de l'objet d'étude, à savoir la culture qui est considérée dans la tradition ontologique comme une totalité, c'est un thème global, qui n'est ni clos ni statique. Rastier (2002a,p. 6) préfère la conception non ontologique de la totalité, «car une culture n'est jamais autarcique et, même dans les conditions d'isolement, se développe à partir d'une histoire partagée». Suivant la méthode praxéologique, l'auteur souligne que les cultures ne peuvent être décrites que différentiellement, à travers les éléments tangibles et réels qui les composent, à savoir les langues et les textes ${ }^{18}$.

Sur le plan épistémologique, les sciences de la culture cherchaient depuis longtemps à se légitimer par l'imitation des méthodes en vigueur dans les sciences de la nature et de la vie, l'anthropologie de C.L.Strauss et la linguistique de Ferdinand de Saussure en sont des exemples significatifs. La synthèse des sciences humaines et des sciences de la nature a donné naissance à des disciplines nouvelles comme la sociobiologie, l'anthropologie néodarwinienne, la biopolitique, la paléoanthropologie, etc. Les sciences de la culture ont également suivi la voie des sciences logico-formelles en proposant des modélisations, observées en l'occurrence en économie et en linguistique. Dans cette synthèse tant évoquée par les sciences de la culture, le problème qui se pose est celui du «voyage» des concepts, des démarches et des théories d'une discipline vers une autre. Souvent, ce sont les savoirs des sciences humaines qui trouvent des difficultés à « intégrer » les concepts et dispositifs méthodiques ${ }^{19}$ relevant des sciences de la nature. Aussi se résignent-elles à suivre la stratégie de l'objectivation et de l'interprétation.

Dans un essai d'extraction des spécificités des sciences de la culture ${ }^{20}$, nous avons constaté que les critères fondamentaux des sciences du texte (Rastier 2001)

\footnotetext{
17 Pour de plus amples informations sur les spécificités des sciences de la culture, cf. El Khattab, 2017 , chap. 1 et 13 .

18 Rastier, (2002a, p. 6).

${ }^{19}$ Cf. Grawitz, (2001,p. 106 et ss.).

20 Cf. EL Khattab, 2017, chap. 13.
} 
sont les mêmes critères que l'auteur aspire à appliquer à l'ensemble des sciences de la culture. Ces critères sont:

(i) la lecture des textes : il s'agit d'une lecture qui s'appuie sur deux principes, le principe philologique et le principe herméneutique. Liée à la sémantique des textes, la lecture productive consiste à mettre en place des sèmes en prenant en considération le contexte. La lecture des textes suivant ce mode interprétatif ${ }^{21}$ permet de caractériser les autres performances sémiotiques ;

(ii) la description, c'est une des spécificités des sciences de la culture qui tendent à décrire les valeurs et non les faits, sans émettre des jugements de valeurs.

(iii) L'étude de la diversité : on entend par là la diversité des langues, des races, des comportements, des rites, etc. Humboldt était l'un des premiers à détourner la recherche en sciences humaines de l'universel vers le particulier.

(iv) L'objectivité du sens : le sens est un niveau d'objectivité qui est analysable en traits sémantiques qui sont des moments stabilisés dans des parcours d'interprétation. Il est tentant d'avancer que toutes les sciences de la culture peuvent être assujetties à ce mode d'interprétation, du fait que la problématique interprétative dépasse les textes et peut s'étendre à d'autres objets culturels comme les images;

(v) Le critère différentiel: la sémiotique des cultures est foncièrement différentielle et comparative ${ }^{22}$, car « une culture ne peut être comprise que d'un point de vue cosmopolitique ou interculturel» ${ }^{23}$.

\footnotetext{
${ }^{21}$ C'est un système interprétatif qui est fondé sur la sémantique textuelle (Rastier, 1989, 2001a) qui se détourne des préoccupations ontologiques de la linguistique. Le sens d'un texte est construit plutôt que donné. Dans ce cas, la signification n'est pas renvoi au monde ; la sémantique développée par l'auteur est une sémantique dé-ontologique, elle s'inscrit dans la tradition de Saussure qui identifie les signifiés en termes de valeur et de différence. Une telle sémantique est décrite ainsi comme étant une sémantique différentielle.

22 La notion de «différence» est empruntée à l'approche structurale, alors que celle de «comparaison» vient de la méthode historique et comparative qui était en vogue au XIXe siècle.

${ }^{23}$ Rastier (2002a, p.5).
} 
Les sciences de la culture tendent à rendre compte des comportements humains en se basant sur des corpus, en ciblant les processus et, dans l'essai d'appréhension des phénomènes et des productions humaines, dont les textes, elles évitent la référence à des ontologies. Ainsi, les sciences de la culture visent selon Rastier (2002a) la diversité culturelle afin de contrecarrer la globalisation des savoirs et suivent la voie des méthodes interprétatives face à la computation des connaissances. Tout ceci montre que ces savoirs méritent d'assumer la fonction fédératrice.

\section{Conclusion}

Nous avons tenté de spécifier les deux notions de «sémiotique des cultures» et celle de «sciences de la culture», nous avons présenté les caractéristiques épistémologiques des deux savoirs, mais aucune de ces expressions ne convient au propos défendu par Rastier. Il s'avère néanmoins que la question du rapport entre les deux types de savoirs est problématique: notre étude a révélé que la disciplinarisation de la sémiotique n'est pas une bonne entreprise et que c'est le programme collectif des sciences de la culture qui compte. Les écrits de Rastier montrent qu'il est fédéraliste, il considère que la sémiotique des cultures est enfermée sur elle-même. Nous estimons que cette dernière n'est pas assez mûre pour concurrencer les sciences de la culture dans sa vocation fédératrice. Il parait que la définition d'une perspective proprement sémiotique sur la culture est un chantier de la recherche dans l'avenir.

\section{Bibliographie}

CASSIRER, E. (1942), Logique des sciences de la culture, trad.fr. 1991, Paris, Cerf.

DILTHEY, W., (1942), Introduction à l'étude des sciences humaines : essai sur le fondement qu'on pourrait donner à l'étude de la société et de l'histoire, PUF.

DILTHEY W., (1947), Le monde de l'esprit, Paris, Aubier (édition originale en langue allemande, 1925).

ECO, U., (1984), Sémiotique et philosophie du langage, PUF.

ECO, U., (1987), « Notes sur la sémiotique de la réception », in Actes sémiotiques, documents, IX, 81, pp.5-27.

ECO, U., (1988), Le signe, éd. Labor, Le livre de poche, biblio essais, LP10.

ECO, U., (1994), Les limites de l'interprétation, le livre de poche. 
FONTANILLE, J., (2004), « Textes, objets, situations et formes de vie : les niveaux de pertinence de la sémiotique des cultures», ms, publié dans Revista dell'Associazione Italian di studia Semiotici, 22 pages.

FONTANILLE, J., (2008), Pratiques sémiotiques, PUF.

FONTANILLE, J., (2013), « La sémiotique est-elle un art ? », ms. 17 pages.

GRAWITZ, M., (2001), Méthodes en sciences sociales, Dalloz.

GREIMAS,A.J.,(1966), Sémantique structurale , Larousse.

GREIMAS,A.J.,(1977), «La sémiotique », in CAUSSAT, P. et al. (1977), La linguistique, collection encyclopédique, Larousse, pp.223-229.

GREIMAS,A.J., et J. COURTES, (1979), Sémiotique. Dictionnaire raisonné de la théorie du langage, Hachette.

GREIMAS,A.J.,(1984), « Entretien réalisé par J. Fontanille », Langue française, nº 61, pp.121-128.

GREIMAS,A.J. et LANDOWSKI, E., (1997), Sémiotique et sciences sociales, Hachette.

RASTIER,F.,(1989) , Sens et textualité, PUF.

RASTIER,F.,(1996), "Problématique du signe et du texte », Intellectica, nº 23, pp. 11-52.

RASTIER, F.,(2001), "Sémiotique et sciences de la culture », Linx, 2001, n 44-45, pp. 149-168.

RASTIER, F.,(2001a), Arts et sciences du texte, PUF.

RASTIER, F. et BOUQUET, S.,(2002), Une introduction aux sciences de la culture, PUF.

RASTIER, F., (2002a), "Pluridisciplinarité et sciences de la culture » in Rastier et Bouquet, éds. Une introduction aux sciences de la culture, Présentation, pp.1-10.

RASTIER, F., (2004), « Sciences de la culture et post-humanité », ms. 22 pages.

RASTIER, F., (2008), «Sémiotique et linguistique du corpus », Signata, ms, 33 pages.

RASTIER, F., (2009a),"L'évolution aujourd'hui : à la croisée de la biologie et des sciences humaines », Actes du colloque des 29, 30 et 31 janvier 2009 à l'Académie royale de Belgique, imprimerie Communications s.p.r.l., Louvain -la-Neuve, pp.231-250.

RASTIER, F., (2010b) «Objets et performances sémiotiques: l'objectivation critique dans les sciences de la culture », colloque sur l'homme sémiotique, Namur, 19-21, in texto, Vol. XVI, nº1. 31 pages

RASTIER, F., (2012) , "Sémantique des textes, concepts et application », in EL KHATTAB, D et I. ZELLOU, (coordination), Sciences du texte et problèmes d'interprétation, Cahiers de la Recherche Scientifique, $\mathrm{n}^{\circ} 8$, Faculté des lettres, Mohammedia, pp. 11-42. 